\title{
O papel da tecnologia na construção do conhecimento
}

\author{
Max Cirino de Mattos ${ }^{1}$
}

\begin{abstract}
RESUMO
O artigo discute a tecnologia como uma extensão importante do ser humano, capaz de ampliar seus horizontes de aprendizagem e relação com o mundo - uma ferramenta que pode ser adaptada continuamente para contribuir no processo de desenvolvimento da humanidade. Após a discussão sobre o processo de comunicação ilustrado por Foskett (1990), o artigo discorre sobre o papel da tecnologia na formação da identidade individual e coletiva. São apresentados alguns estudos em andamento para demonstrar que as possibilidades tecnológicas, no sentido aqui abordado, são praticamente infinitas - assim como as implicações éticas e a necessidade de estudos aprofundados sobre tais questões.
\end{abstract}

Palavras-chave: Tecnologia. Comunicação. Identidade. Conhecimento.

\section{INTRODUÇÃO}

O avanço das tecnologias de informação e os custos envolvidos na instalação e manutenção de sistemas de processamento de dados são temas recorrentes nos dias de hoje. Podemos atribuir esses custos elevados a três fatores: as limitações de capacidade de armazenamento e processamento das máquinas - mesmo que as máquinas atuais sejam muitas vezes mais eficientes que as de cinco anos atrás; a dificuldade de interpretação das linguagens do computador - que exigem pessoal especializado; e, finalmente, a dificuldade de especificação das necessidades informacionais de um determinado programa (ou sistema).

A única razão para iniciar este tópico com um parágrafo tão óbvio é que se pretende contribuir para a desmistificação dessa tendência tecnoutópica pouco reflexiva que parece sufocar em parte o desenvolvimento científico atual. As ideias apresentadas no parágrafo anterior já eram discutidas, por exemplo, por McGee em 1959.

Em outro trecho, que pode perfeitamente se referir aos dias atuais, o autor diz que "enquanto muitas vezes maior e mais rápido que os seus homólogos de cinco anos atrás, as máquinas atuais de processamento de dados ainda têm velocidade e capacidade limitadas" (McGEE, 1959, p. 2, tradução do autor). Em síntese: essa velocidade - e paradoxal limitação - do desenvolvimento tecnológico computacional é uma constante histórica, e não um fato recente e extraordinário.

De acordo com Alvarenga Neto (2008), organizações modernas focam seus investimentos em tecnologia; no entanto, sem muita atenção na informação, nas pessoas e nos seus conhecimentos. 0 autor ainda afirma que a limitação de uma perspectiva tecnológica consiste em um foco muito direcionado às informações produzidas internamente pelas organizações - o que é importante, mas não suficiente - e em fontes de informação baseadas em computadores.

Para o desenvolvimento deste trabalho, considera-se a tecnologia como uma ferramenta que pode ser adaptada continuamente para contribuir no processo de desenvolvimento da humanidade. Nesse sentido, ela 
não é tratada como um fator de nivelamento, restrição ou limitação, mas, ao contrário, como uma extensão do próprio ser que a utiliza de forma cada vez mais personalizada.

De acordo com Virgil (2008, p. 56),

uma pessoa, ao estender as suas capacidades por meio de uma tecnologia, é parcialmente condicionada por ela. Ao mesmo tempo, a pessoa passa a ter ao seu dispor um conjunto infinito de possibilidades e configurações para as suas habilidades.

O autor sintetiza essa afirmação na Figura 1.

Figura 1 - 0 ser humano e a tecnologia

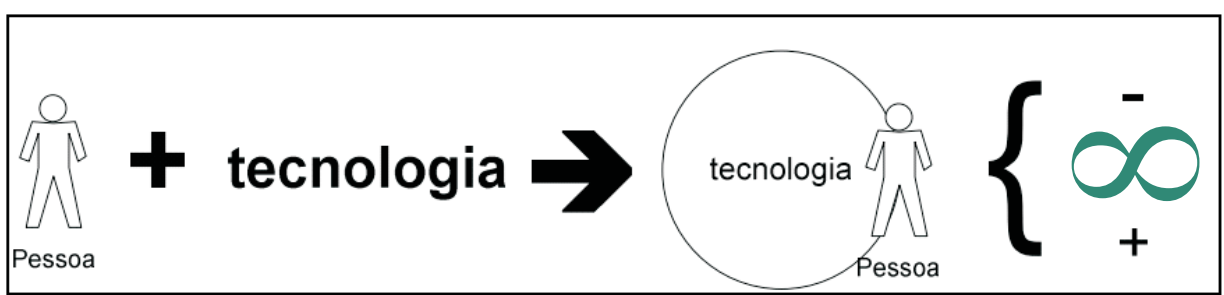

Fonte: Virgil (2008)

Dessa forma, as possibilidades de expressão são potencialmente infinitas, e a própria linguagem passa por uma transformação estrutural, uma vez que cada indivíduo adquire maior liberdade para se expressar sem a necessidade de se identificar - o que pode, simultaneamente, comprometer a capacidade de interação e coordenação das atividades da espécie. A relação com o outro e com o próprio mundo passa a ser construída de forma cada vez mais pessoal, e os símbolos, signos e o próprio discurso assumem características distintas em virtude da liberdade de expressão e criação adquiridas pelo homem. A transição para a formação de um novo meio de coordenação das relações individuais iniciou-se com as modernas tecnologias de comunicação, e não é possível afirmar se vai se consolidar em uma nova forma ou se estará continuamente mudando.

Sob esse aspecto, uma das sugestões apontadas por Mattos (1998) refere-se ao estudo da relação entre o volume muito grande de informações dinâmicas recebidas em um ambiente globalizado e a limitação cognitiva individual para processar tal conteúdo.

O dinamismo do ambiente globalizado representa um processo de mudança contínua e de inovações, enquanto a utilização de paradigmas que nos permitem reduzir a variedade de estímulos recebidos (entre eles, o volume de informações) relaciona-se a certa estabilidade. Dado que a adoção de um paradigma demanda certo tempo, e que, simultaneamente, as mudanças contínuas sugerem a quebra de paradigmas, como filtrar os estímulos recebidos, expandindo-se a capacidade do aparelho psíquico ou criando-se estruturas abstratas flexíveis? (MATTOS, 1998, p. 115, grifo nosso).

Castells (2000, p. 39) explora essa relação dialética entre o individual e o social (globalizado):

A questão principal, em um mundo caracterizado pela globalização e fragmentação simultâneas, vem a ser esta: 'Como combinar novas tecnologias e memória coletiva, ciência universal e culturas comunitárias, paixão e razão?'. E por que observamos a tendência oposta em todo o mundo, ou seja, a distância crescente entre globalização e identidade, entre a Rede e o Ser?

Essa proposição de Ser e Rede - individual e coletivo - também observada na leitura de Capra (1999) possibilitou uma expansão da ideia inicial discutida na dissertação, que se limitava ao estudo de organizações empresariais - o horizonte de pesquisa foi ampliado para uma relação entre o indivíduo e os grupos sociais, independentemente de estes serem organizações empresariais.

Com o propósito de abordar-se a inserção do indivíduo em grupos sociais e as interações provenientes dessa relação, tornou-se oportuna a adoção dos conceitos elaborados por Maturana e Varela (1995), principalmente aqueles relacionados aos fenômenos sociais e aos organismos e sociedades. Os autores afirmam que "a existência de um sistema social humano requer a plasticidade operacional (comportamental) de seus componentes” (MATURANA; VARELA, 1995, p. 224). De acordo com os autores, os indivíduos, como 
componentes de sistemas sociais, operam por meio da linguagem na construção e manutenção desses sistemas, utilizando-se do processo de comunicação.

\section{A IMPORTÂNCIA DO PROCESSO DE COMUNICAÇÃO}

Foskett (1990) apresenta uma proposta de estruturação na forma de pirâmide para a definição de alguns termos envolvidos no processo de comunicação cuja lógica é interessante para o desenvolvimento deste texto. A Figura 2 representa os principais componentes propostos pelo autor. Conforme detalhamento adiante, os fenômenos ocorrem na natureza, enquanto dado, informação e conhecimento são conceitos usados em um nível individual, e o saber é coletivo - produzido socialmente.

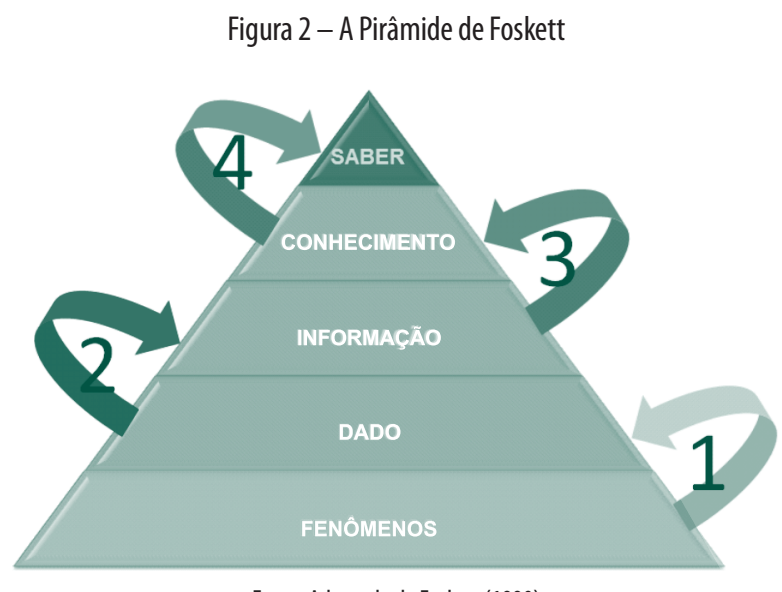

Fonte: Adaptado de Foskett (1990)

O autor apresenta como base da pirâmide o ambiente onde acontecem todos os fenômenos, fonte inesgotável das experiências resultantes da interação do homem com a natureza. A partir do contato com esse ambiente, o homem experimenta sensações e percepções que apresentam o potencial de se transformarem em conceitos com algum significado, ou dados.

Dado, conforme o autor é o elemento mais básico que a mente humana pode trabalhar e com base no qual pode gerar informação. Fatos simples, atômicos, como as propriedades dos materiais, as datas de aniversários, localização de cidades e países são dados com formato linguístico e podem apenas ser apresentados como verdade, verificados pela investigação, e tidos como universais.

Conforme salienta o autor, a informação consiste em conjuntos de dados organizados na mente humana com base em algum critério estruturado - a informação pode ser considerada a contextualização do dado, dotando-o de um significado pertencente a um sistema específico de interpretação. Essa operação ocorre, de acordo com o autor, no nível individual. Esse sistema de interpretação é resultante de uma série de variáveis, entre elas a experiência prática, e as sensações são então transformadas em pensamentos estruturados, e as percepções em conceitos - e justamente porque cada indivíduo possui uma experiência única de vida, seu conhecimento também é único.

A distinção entre informação e conhecimento é concebida pelo autor a partir desta característica individual - o conhecimento não é simplesmente um estoque de informações, pois cada pessoa interpretou, analisou e absorveu as informações de formas diferentes de acordo com sua vivência, seu repertório. Em resumo, essa transformação de informações em conhecimento depende de ações de integração intelectual.

O saber é tratado pelo autor como o conhecimento coletivo de uma comunidade, derivado da vida em sociedade e das diversas trocas de experiências do grupo. Ele consiste da busca de teorias mais genéricas sobre o mundo, mais amplas como a Teoria Geral dos Sistemas - mas com a preocupação em um processo de comunicação global eficiente.

Foskett (1990) apresenta, em síntese, uma forma de estruturação de fenômeno-dado-informaçãoconhecimento-saber que se desenvolve a partir dos processos de investigação-organização-assimilação- 
compartilhamento - e como cadeia de comunicação pode ser aplicada de forma ampla aos conhecimentos humanos.

\section{ATECNOLOGIAEA COMUNICAÇÃO}

A comunicação também é um dos principais focos do desenvolvimento tecnológico atual. A partir da evolução e diversificação dos meios de comunicação (meios tecnológicos), são oferecidas novas formas de relacionamento entre indivíduos e grupos sociais, ou seja, de acordo com os conceitos de Maturana e Varela (1995), ampliamos as possibilidades de coordenações comportamentais resultantes das condutas comunicativas dos acoplamentos sociais.

Em um processo de realimentação, novas coordenações comportamentais podem gerar novos desenvolvimentos tecnológicos, que por sua vez ampliam as possibilidades de novas coordenações comportamentais. Castells (2000) lembra que esse processo gera um ciclo no qual a aplicação da tecnologia torna-se elemento essencial no desenvolvimento social.

Sem dúvida, a habilidade ou inabilidade de as sociedades dominarem a tecnologia e, em especial, aquelas tecnologias que são estrategicamente decisivas em cada período histórico, traça seu destino a ponto de podermos dizer que, embora não determine a evolução histórica e a transformação social, a tecnologia (ou sua falta) incorpora a capacidade de transformação das sociedades, bem como os usos que as sociedades, sempre, em um processo conflituoso, decidem dar ao seu potencial tecnológico (CASTELLS, 2000, p. 26).

Esse processo pode ser representado conforme a Figura 3.

Figura 3 - Desenvolvimento tecnológico e coordenações comportamentais

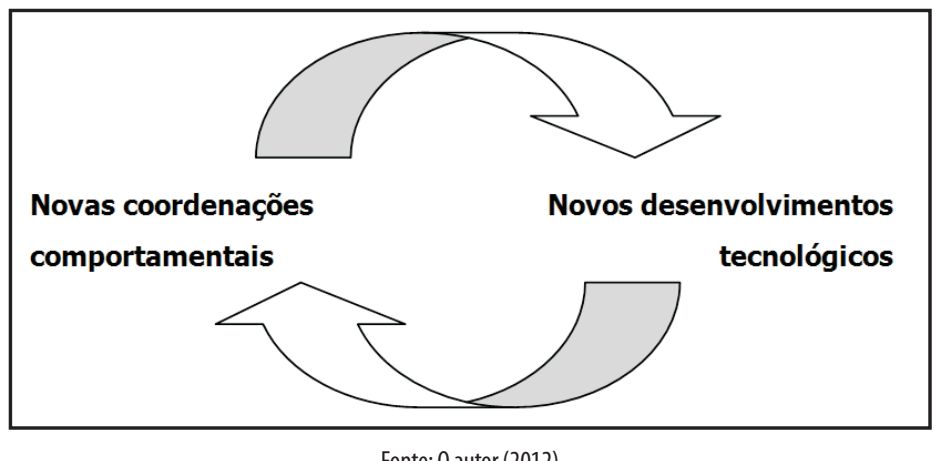

É importante ressaltar que a tecnologia não determina a sociedade, nem a sociedade determina a tecnologia, mas existe uma relação sinérgica entre as duas. Essa relação tem um ponto central que é a utilização da tecnologia - somente por intermédio de seu uso prático ela pode proporcionar novas formas de comunicação, potencializando uma transformação social que poderá exigir um novo desenvolvimento tecnológico.

\footnotetext{
O que caracteriza a atual revolução tecnológica não é a centralidade de conhecimentos e informação, mas a aplicação desses conhecimentos e dessa informação para a geração de conhecimentos e de dispositivos de processamento/comunicação da informação, em um ciclo de realimentação cumulativo entre a inovação e seu uso. [...] 0 ciclo de realimentação entre a introdução de uma nova tecnologia, seus usos e seus desenvolvimentos em novos domínios torna-se muito mais rápido no novo paradigma tecnológico. Conseqüentemente, a difusão da tecnologia amplifica seu poder de forma infinita, à medida que os usuários apropriam-se dela e a redefinem (CASTELLS, 2000, p. 50-51).
}

Essa alta velocidade de desenvolvimento tecnológico implica um processo acelerado de disponibilização de novas formas de acoplamento, que podem levar o indivíduo a uma diversidade enorme de opções entre as quais ele precisa escolher a(s) que lhe permita(m) manter sua adaptação contínua ao meio. 
Esse paradoxo entre a estabilidade individual e a mudança contínua do meio, considerando-se a necessidade de acoplamento estrutural do indivíduo para sua sobrevivência, torna-se fundamental para a compreensão da relação entre o individual e o coletivo.

A identidade individual está se tornando a principal e, às vezes, única fonte de significado em um período histórico caracterizado pela ampla desestruturação das organizações, deslegitimação das instituições, enfraquecimento de importantes movimentos sociais e expressões culturais efêmeras (CASTELLS, 2000, p. 23)

A nova estrutura dos agrupamentos sociais em forma de Rede - de acordo com o conceito de Castells - e a ampliação da capacidade individual de utilização criativa dos meios de comunicação geram um contexto no qual a diversificação de opções de coordenações comportamentais acaba por fragmentar grupos sociais e estruturas culturais - componentes da identidade coletiva - que serviam de base para a identidade individual. Nesse sentido, o desenvolvimento da identidade individual tendo por base o uso criativo de novas tecnologias de comunicação gera a fragmentação da identidade coletiva, ao mesmo tempo em que depende dela. Segundo Castells (2000, p. 51), esse uso criativo é um ponto muito importante uma vez que se

segue uma relação muito próxima entre os processos sociais de criação e manipulação de símbolos (a cultura da sociedade) e a capacidade de produzir e distribuir bens e serviços (as forças produtivas). Pela primeira vez na história, a mente humana é uma força direta de produção, não apenas um elemento decisivo no processo produtivo.

Além disso, o autor também cita a formação da identidade coletiva tomando por base as coordenações comportamentais individuais. De acordo com ele,

a comunicação simbólica entre os seres humanos e o relacionamento entre esses e a natureza, com base na produção (e seu complemento, o consumo), experiência e poder, cristalizam-se ao longo da história em territórios específicos, e assim geram culturas e identidades coletivas (CASTELLS, 2000, p. 33).

A partir desse outro processo cíclico, no qual identidade individual e identidade coletiva se modificam continuamente com base na comunicação (coordenações comportamentais), e considerando a discussão anterior sobre desenvolvimento e aplicação de inovações tecnológicas, percebe-se que a participação da tecnologia é fundamental na análise dessa relação.

A Figura 4 apresenta o papel da tecnologia nas relações que podem ocorrer entre a identidade individual e a coletiva, considerando-se as relações de produção, experiência e poder determinantes da organização das sociedades. Fundamentando-se nessa noção, novos desenvolvimentos tecnológicos, como foi recentemente o caso das redes sociais, estarão conformados no ciclo de interações (Figura 4).

Figura 4 - Identidade individual, identidade coletiva e as novas tecnologias

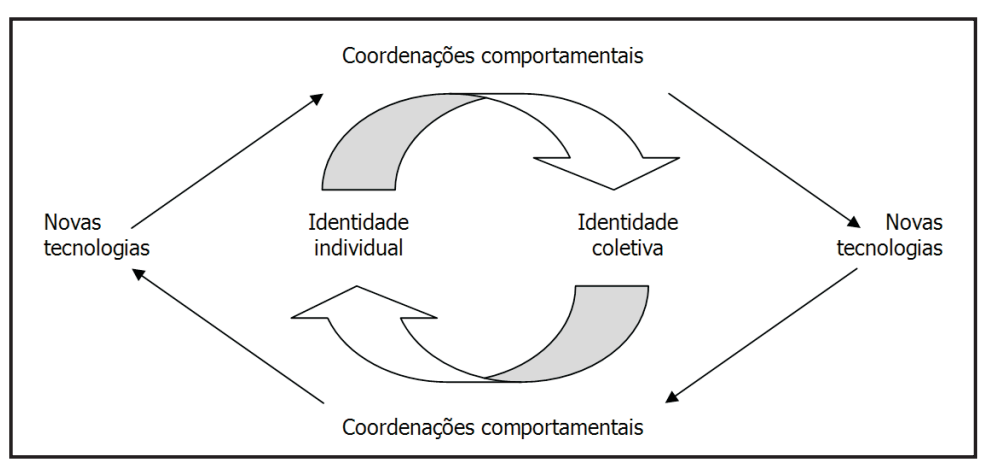

Fonte: 0 autor (2012)

Também são importantes alguns pontos identificados por Alvarenga Neto com base nas considerações de Castells (2000) sobre o novo paradigma tecnológico: se antes se tratava de informações para agir sobre a tecnologia, agora são tecnologias para agir sobre a informação; a informação é parte integral de toda atividade 
humana, e a tecnologia molda todos os processos de existência individual ou coletiva; um contexto de interação e estruturação do não estruturado, mantendo a sua flexibilidade relaciona-se à lógica de redes; e a convergência de tecnologias para sistemas altamente integrados (ALVARENGA NETO, 2008).

É importante destacar que, em se tratando de novas tecnologias - além dessa diversidade de aplicações tecnológicas de cunho mais comercial que são amplamente divulgadas -, existem inúmeros estudos científicos que superam a noção ingênua de que a tecnologia apenas torna processos mais eficientes. Não apenas em relação às questões de poder, conforme Virgil (2008, p. 58):

Não menos importante, o poder também está relacionado à esfera humana da tecnologia. Ausência de acesso básico à tecnologia, seleção de uma tecnologia em detrimento de outra, obrigatoriedade de uso, todas são facetas que podem denotar o exercício arbitrário do poder.

Exemplos recentes de pesquisas, importantes para o conceito de tecnologia que se pretende trabalhar, incluem os trabalhos de Nicolelis (2011), Warwick (2010), Warwick e Ruiz (2008), Maharbiz e Sato (2010), e Turkle $(2005,2008,2011)$. Os impactos futuros dessas pesquisas são inimagináveis, mas não devem alterar o fluxo proposto na figura anterior.

Miguel Angelo Laporta Nicolelis, internacionalmente reconhecido neurocientista brasileiro, ressalta no próprio título de seu recente livro - Muito além do nosso eu: a nova neurociência que une cérebro e máquinas, e como ela pode mudar nossas vidas - a importância e o avanço científico atual na exploração do impacto social dessa relação homem-máquina. 0 autor apresenta o conceito de Interface Cérebro-Máquina (ICM) e algumas de suas aplicações para a medicina. Expandindo as possibilidades de uso dessas interfaces ele ainda afirma que,

\begin{abstract}
surpreendentemente, as futuras aplicações de ICMs prometem avançar muito além dos limites da medicina. Em algumas gerações, seu desenvolvimento tecnológico capacitará nossos descendentes a realizar ações e experimentar sensações que poucos seriam capazes de imaginar ou mesmo verbalizar hoje em dia. As ICMs podem transformar a maneira como interagimos com as ferramentas que fabricamos para dialogar com o mundo que nos cerca, a forma como nos comunicamos uns com os outros, bem como nossa interação com ambientes distantes e outros mundos (NICOLELIS, 2011, p. 24).
\end{abstract}

Warwick (2010) discute as implicações éticas e filosóficas de uma de suas recentes pesquisas: robôs com cérebros biológicos. Renomado pesquisador na área de inteligência artificial, robótica e engenharia biomédica, realizou uma cirurgia em 1998 para implantar um dispositivo eletrônico em seu braço, como parte de suas pesquisas, tornando-se o primeiro humano com um chip implantado. Em uma de suas pesquisas, foi capaz de movimentar um braço mecânico a $55.000 \mathrm{~km}$ de distância com o movimento de seu braço, transmitindo os sinais emitidos pelo referido chip pela internet. Suas pesquisas apresentam contribuições significativas para a integração entre humanos e robôs, além de discutirem a possibilidade de existência de organismos híbridos (homem-máquina).

Maharbiz e Sato (2010) igualmente apontam para a possibilidade de organismos híbridos e descrevem "insetos mecânicos" até quatro vezes mais leves que uma mosca, desenvolvidos no Harvard Microrobotics Laboratory. Esse é apenas um entre os vários exemplos de miniaturização dos dispositivos tecnológicos que lhes permitiram o controle do voo de um besouro.

Importantes pesquisas sobre a interação entre homens e máquinas também são desenvolvidas por Sherry Turkle, fundadora do MIT Initiative on Technology and Self que escreve sobre o "lado subjetivo" do relacionamento das pessoas com a tecnologia.

\title{
4CONCLUSÃO
}

0 artigo avaliou a tecnologia com base na perspectiva de que a velocidade - e paradoxal limitação - do desenvolvimento tecnológico computacional é uma constante histórica, e não um fato recente e extraordinário.

A tecnologia foi apresentada como uma extensão do próprio ser humano, que a utiliza de forma cada vez mais personalizada no seu processo de comunicação e na interação entre o indivíduo e seu meio social.

Nessa relação entre o individual e o social, ressaltou-se que a tecnologia não determina a sociedade, nem a sociedade determina a tecnologia, mas existe uma relação sinérgica entre as duas, na qual o ponto central é a 
utilização da tecnologia: somente mediante seu uso prático ela pode proporcionar novas formas de comunicação, potencializando uma transformação social que poderá exigir um novo desenvolvimento tecnológico, em um processo cíclico.

Foi destacada a importância e o avanço científico atual na exploração do impacto social da relação homemmáquina baseado na apresentação de alguns estudos relacionados a robôs com cérebros biológicos, organismos híbridos e o lado subjetivo do relacionamento das pessoas com a tecnologia.

É possível perceber, com esses poucos exemplos citados diante das inúmeras pesquisas e avanços científicos em andamento, que as possibilidades tecnológicas, no sentido aqui abordado, são praticamente infinitas - assim como as implicações éticas e a necessidade de estudos aprofundados sobre essas questões.

\section{THE ROLE OF TECHNOLOGY IN THE CONSTRUCTION OF KNOWLEDGE}

\section{ABSTRACT}

The paper discusses technology as an important extension of the human being.It is able to broaden their horizons and their learning about the world - a tool that can be adapted to continuously contribute to the development of humanity. After discussing the communication process illustrated by Foskett (1990) the paper discusses the role of technology in shaping individual and collective identity. Some ongoing studies are described to demonstrate that the technological possibilities - in the sense discussed here - are practically endless as are the ethical implications and the need for further studies on these issues.

Keywords: Technology. Communication. Identity. Knowledge.

\section{REFERÊNCIAS}

ALVARENGA NETO, Rivadávia Drummond de. Gestão do Conhecimento em Organizações: proposta de mapeamento conceitual integrativo. Belo Horizonte: Saraiva, 2008.

CAPRA, Fritjof. A teia da vida: uma nova compreensão científica dos seres vivos. São Paulo: Cultrix, 1999.

CASTELLS, Manuel. A sociedade em rede. 3. ed. São Paulo: Paz e Terra, 2000.

FOSKETT, D. J. The communication chain. In: MIKHAILOV, Aleksandr Inovich; FOSKETT, D.J. The information environment: a world view. North-Holland: Elsevier Science Publischers, 1990. p. 177-183.

MAHARBIZ, M. M.; SATO, H. Cyborg Beetles: Tiny flying robots that are part machine and part insect may one day save lives in wars and disasters. Scientific American, v. 303, n. 6, p. 94-99, 2010.

MATTOS, Max Cirino de. As informações que dão suporte ao processo decisório e a influência dos grupos que as processam: um estudo de caso em uma empresa do setor público de telecomunicações. 1998. Dissertação (Mestrado em Ciência da Informação)-Universidade Federal de Minas Gerais, Belo Horizonte, 1990.

MATURANA, Humberto; VARELA, Francisco. A árvore do conhecimento: as bases biológicas do entendimento humano. Campinas: Editora Psy, 1995.

McGEE, William. C. Generalization: Key to Successful Electronic Data Processing. J. ACM, [S.I.], v. 6, n. 1, p. 1-23, 1959.

NICOLELIS, Miguel Angelo Laporta. Muito além do nosso eu: a nova neurociência que une cérebro e máquinas e como ela pode mudar nossas vidas. São Paulo: Companhia das Letras, 2011.

TURKLE, Sherry. The Second Self: Computers and the Human Spirit. London: The MIT Press, 2005.

TURKLE, Sherry. The Inner History of Devices. London: The MIT Press, 2008. 